\title{
Multiplanar 4D strain analysis with spatial mapping to 3D LGE quantification: relationships in chronic Ischemic Cardiomyopathy
}

\author{
Alessandro Satriano ${ }^{1,2^{*}}$, Vijay Kandalam ${ }^{1}$, Yoko Mikami ${ }^{1}$, Nita Guron ${ }^{3}$, Hanna Medwid ${ }^{4}$, Bobby Heydari ${ }^{1,3}$, \\ Naeem Merchant ${ }^{1,3}$, Andrew G Howarth ${ }^{1,2}$, Carmen Lydell ${ }^{1,3}$, Teresa A Whitman ${ }^{5}$, Maria Drangova ${ }^{7}$, Raymond Yee ${ }^{6}$, \\ James A White ${ }^{1,2}$
}

From 18th Annual SCMR Scientific Sessions

Nice, France. 4-7 February 2015

\section{Background}

Myocardial strain analysis has been proposed as a surrogate for regional replacement fibrosis (scar) in patients with ischemic cardiomyopathy (ICM). However, contractile function is often degraded in non-scarred tissue, conceivably due to a composite of interstitial fibrosis, metabolic aberrations and abnormal electro-mechanical coupling. We tested a novel 4D strain analysis tool to examine strain characteristics of scarred and nonscarred myocardium in patients with advanced ICM.

\section{Methods}

Nineteen patients with ICM and 10 healthy controls were studied. Cine and Late Gadolinium Enhancement (LGE) imaging was performed using 3.0T MRI. LV signal threshold-based ( $>6 \mathrm{SD})$ \%LGE maps were obtained using cvi42 (Circle Cardiovascular Inc., Calgary, Canada). 4D strain analysis (Figure 1) was performed using novel prototype software employing a 4D displacement field, providing spatially matched Green-Lagrange $2^{\text {nd }}$ principal, radial, circumferential and longitudinal strain maps. \%LGE and strain were co-registered to a 72-segment model.

\section{Results}

Mean age of ICM patients was $72.3 \pm 6.8$ years with LVEF of $26.5 \pm 7.7 \%$. Among 1368 analyzed segments, 823 had no LGE(<5\%), 299 had 5-50\%LGE, 246 had LGE $\geq 50 \%$ (transmural). Mean age of healthy controls

TStephenson Cardiac Imaging Centre, Libin Cardiovascular Institute, Calgary, $A B$, Canada

Full list of author information is available at the end of the article was $28.2 \pm 7.5$ years with LVEF of $61.8 \pm 7.4 \%$, all segments with no LGE. Segmental strain analysis using all 4 metrics showed substantial reductions in mean peak amplitude for ICM segments without LGE versus healthy controls $(\mathrm{p}<0.05)$. Within the ICM cohort, LGE $\geq 50 \%$ segments showed reduced strain amplitudes versus segments without LGE (mean reduction 29.0 $\pm 13.6 \%$ - Figure 2$)$ for all strain metrics $(\mathrm{p}<0.05)$. Significant difference was found between $\mathrm{LGE}<50 \%$ and LGE $\geq 50 \%$ segments. ROC analysis identified AUCs for detection of $\mathrm{LGE} \geq 50 \%$ of $0.63,0.28,0.62$, and 0.62 , respectively. Using optimal cut-offs, corresponding sensitivity was $59.8 \%, 32.5 \%, 58.5 \%$, and $57.7 \%$, while specificity was 59.1, 32.3\%, 58.2 and $57.8 \%$. AUCs for identifying viable $(\mathrm{LGE}<50 \%)$ segments were $0.37,0.72$, 0.38 and 0.38 , the greatest sensitivity and specificity being $68.5 \%$ and $67.5 \%$, respectively, for Radial Strain. The PPV and NPV achieved for identifying a viable segment were $90.6 \%$ and $32.0 \%$, respectively.

\section{Conclusions}

In chronic ICM, spatially matched 4D strain/LGE analysis identifies reduced strains in scarred segments, however also significant pathology in remote tissue compared to healthy controls. The latter limits the NPV of strain analysis for identifying non-scarred segments. However, this study demonstrates a novel capacity of CMR-based strain quantification to characterize the global health of remote tissue. As such, this provides a novel imaging marker for the quantification of remote tissue remodeling / functional integrity and warrants investigation for its prognostic value in ICM. 


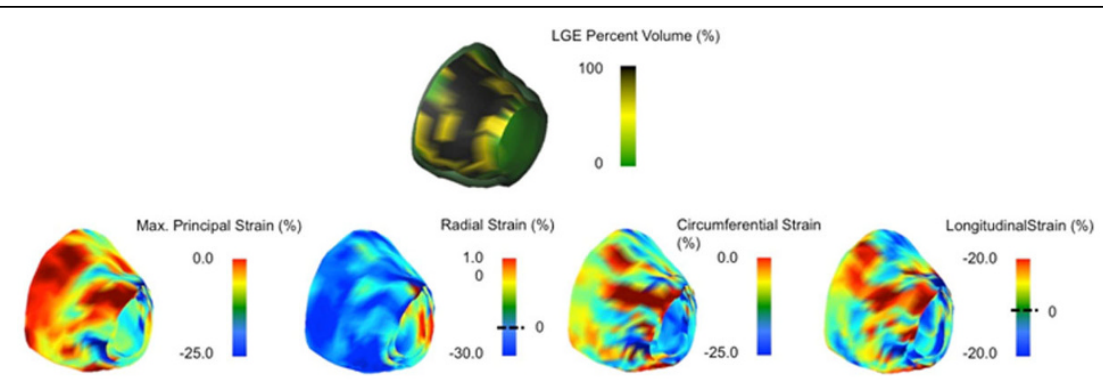

Figure 1 Scar and strain LV 3D distribution.Top Pane: Distribution of Late Gadolinium Enhancement (Relative Enhanced Aread) across the endocardial LV surface. Bottom Pane: Peak-Systolic 3D distribution of Maximum Principal, Radial, Circumferential and Longitudinal Strain.

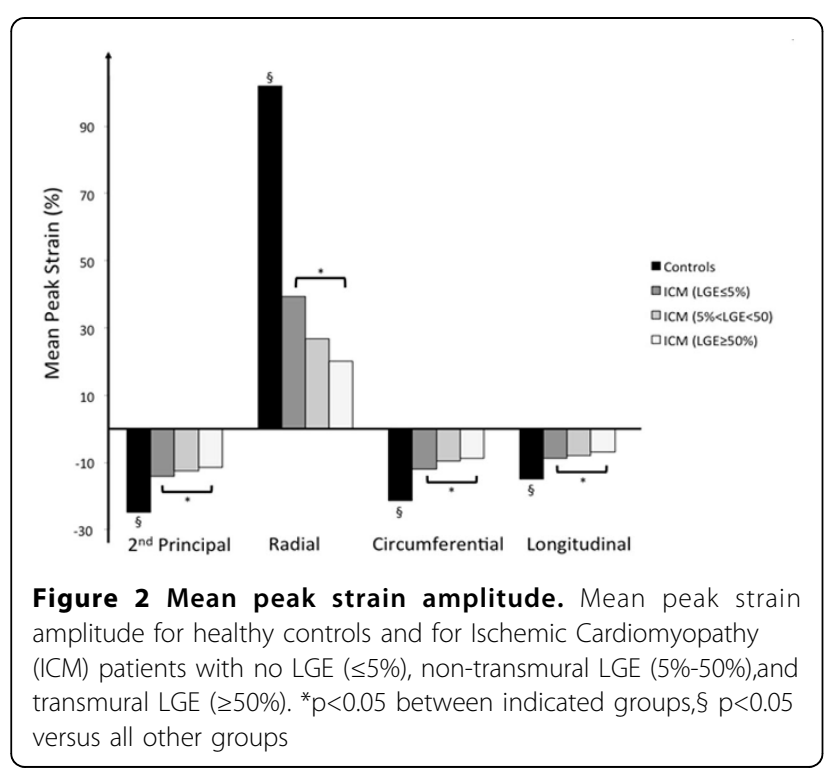

\section{Funding}

Dr. Satriano receives support from Mitacs Canada and Medtronic of Canada, Ltd. Dr. White is supported by a New Investigator Award from Alberta Heart and Stroke Foundation.

\section{Authors' details}

${ }^{1}$ Stephenson Cardiac Imaging Centre, Libin Cardiovascular Institute, Calgary, $A B$, Canada. ${ }^{2}$ Division of Cardiology, Department of Medicine, University of Calgary, Calgary, AB, Canada. ${ }^{3}$ Department of Diagnostic Imaging, University of Calgary, Calgary, AB, Canada. ${ }^{4}$ Queen's University, Kingston, ON, Canada. ${ }^{5}$ Medtronic, Inc., Minneapolis, MN, USA. ${ }^{6}$ Department of Medicine, Western University, London, ON, Canada. ${ }^{7}$ Robarts Research Institute, Western University, London, ON, Canada.

Published: 3 February 2015

\section{doi:10.1186/1532-429X-17-S1-P33}

Cite this article as: Satriano et al:: Multiplanar 4D strain analysis with spatial mapping to 3D LGE quantification: relationships in chronic Ischemic Cardiomyopathy. Journal of Cardiovascular Magnetic Resonance 2015 17(Suppl 1):P33

\section{Submit your next manuscript to BioMed Central} and take full advantage of:

- Convenient online submission

- Thorough peer review

- No space constraints or color figure charges

- Immediate publication on acceptance

- Inclusion in PubMed, CAS, Scopus and Google Scholar

- Research which is freely available for redistribution 